\title{
Genetic susceptibility and alterations in relation to arsenic exposure, metabolism and toxicity
}

\author{
H. Ahsan \& B. Pierce \\ Institute for Population and Precision Health, Chicago Center for Health and Environment, \\ The University of Chicago, Chicago, IL, USA
}

\begin{abstract}
Chronic arsenic exposure increases risks for mortality and morbidity in humans. In addition to dose, duration of exposure and other host factors, the risks vary based on genetic status of individuals. We undertook some of the largest and most comprehensive genome-wide investigations of genetic susceptibility to metabolism and health effects of arsenic. Two major genetic loci have been identified to play significant roles: arsenic methyl-transferase gene on chromosome 10 and formiminotransferase cyclodeaminase gene on chromosome 21. Candidate gene studies found genes in the oxidative stress, DNA repair and immune pathways also to play roles. Our genome-wide investigations of gene expression and epigenetic variations identified novel genomic alterations induced by arsenic exposure in human. The presentation at the conference will integrate these findings, both published and unpublished, along with other recent key genomic investigations of arsenic metabolism and toxicity in different populations in the world.
\end{abstract}

\section{INTRODUCTION}

Arsenic contamination of drinking water is a major public health problem affecting $>100$ million people in many countries, increasing their risk for a wide array of diseases and mortality. Specific diseases liked with arsenic exposure include skin lesions, cancer, diabetes, cardiovascular disease, non-malignant lung disease, and overall mortality. There is interindividual variation in arsenic metabolism efficiency and susceptibility to arsenic toxicity; however, the basis of this variation is not well understood. Once absorbed into the blood, most inorganic arsenic (iAs) is converted to mono-methylated (MMA) and then di-methylated (DMA) forms, facilitating excretion in urine. Arsenic metabolism is influenced by lifestyle and demographic factors, as well as inherited genetic variation. Here we focus on the genetic susceptibility to arsenic metabolism and toxicity and associated mechanisms and molecular alterations.

\section{METHODS}

We conducted the largest and most comprehensive genomic investigations of arsenic metabolism and toxicity to date, through a series of genome-wide and epigenome-wide association studies and molecular genomic studies involving gene expression and methylation. GWAS studies of common variants as well as whole-exome study involving nonsynonymous, protein-coding variations were conducted in relation to arsenic metabolism efficiency and toxicity phenotypes. These studies were conducted within two large cohorts: Health Effects of Arsenic Longitudinal Study (HEALS) and Bangladesh vitamin E and Selenium Trial (BEST). Because variants influencing arsenic metabolism may alter susceptibility to arsenic toxicity, we also investigated the roles of metabolite-associated SNPs in arsenic-induced premalignant skin lesions, the hallmark of chronic arsenic toxicity.

Genomic DNA and RNA from individuals participating in HEALS and BEST were utilized for SNP array, exome array, gene expression array and methylation array to generate data on common variants, exomic rare variants, mRNA expression and $\mathrm{CpG}$ methylation variations, respectively. The SNP variants were imputed to $1 \mathrm{KG}$ dataset to expand genomic coverage. Arsenic metabolites in urine were separated using high-performance liquid chromatography and detected using inductively coupled plasma-mass spectrometry, as previously described (Ahsan et al., 2007). Percentages of iAs, MMA and DMA were calculated after subtracting asenobetaine and arsenocholine from total arsenic.

For association analyses, GEMMA (Genome-wide Efficient Mixed Model Association) was used to account for cryptic relatedness, as a substantial number of our participants have a close relative pair in the study. Allele frequencies and linkage disequilibrium (LD) patterns were examined using LDlink and the Geography of Genetic Variants browser. Gene expression and methylation analyses used Illumina software for calling and a number of different specialized software for specific statistical analyses. 


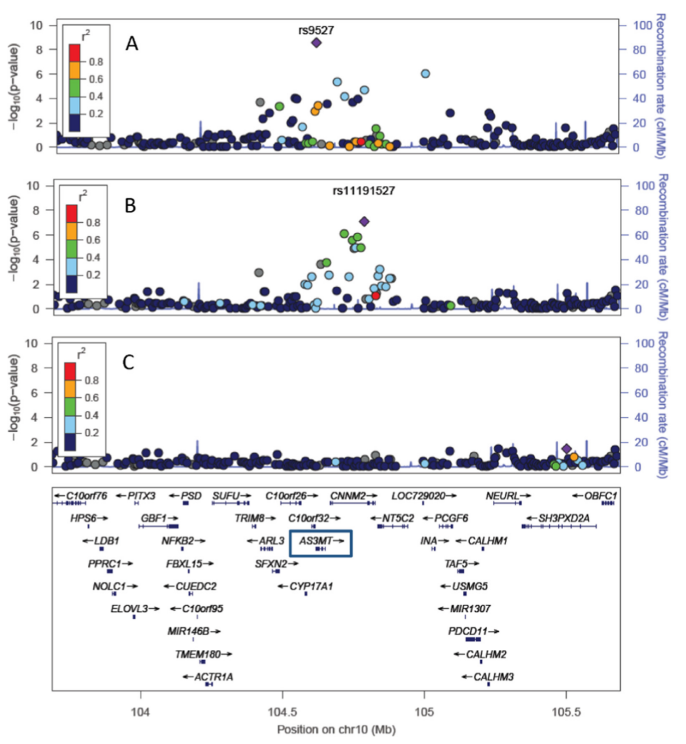

Figure 1. 10q24.32 region variants showing independent associations with DMA\%. A: Overall association; B: Adjusted for rs9527; C: Adjusted for rs9527 and rs11191527.

\section{RESULTS AND DISCUSSION}

In the first genome-wide association study of arsenicrelated metabolism and toxicity phenotypes we previously identified genetic variation near $A S 3 M T$ (arsenite methyltransferase; 10q24.32; $\mathrm{P}<5 \times 10^{-8}$ ) to modify arsenic metabolism efficiency and toxicity (Pierce et al., 2012). We reported two AS3MT genetic variants showing independent associations with arsenic metabolism (Fig. 1) and one of these five variants (rs9527) was also associated with arsenical skin lesion risk $(\mathrm{P}=0.0005)$. The rs 9527 variant was also found to interact with arsenic to influence incident arsenical skin lesion risk $(\mathrm{P}=0.01)$. Expression quantitative trait locus (eQTL) analyses of genomewide expression data from based on lymphocyte RNA suggested that several of our lead variants represent cis-eQTLs for AS3MT $\left(\mathrm{P}=10^{-12}\right)$ and neighboring gene $\mathrm{C} 10$ orf32 $\left(\mathrm{P}=10^{-44}\right)$, which are involved in C10orf32-AS3MT read-through transcription. For our five lead SNPs, we found the rs9527 allele associated with decreased DMA\% and increased skin lesion risk $(\mathrm{P}=0.0005)$, consistent with the hypothesis that DMA is less toxic than MMA.

In a follow-up investigation (Fig. 2), we used a complementary statistical approach to document that two arsenic-methylated species (MMA\% and DMA\%) involve associations with distinct 10q24.32 variants (Jansen et al., CEBP 2016).

To identify additional arsenic metabolism loci, we measured protein-coding variants across the human exome for nearly 5,000 HEALS and BEST individuals. Among 19,992 coding variants genome-wide, the minor allele (A) of rs61735836 was associated with increased urinary iAs\% $\left(\mathrm{P}=2 \times 10^{-12}\right)$
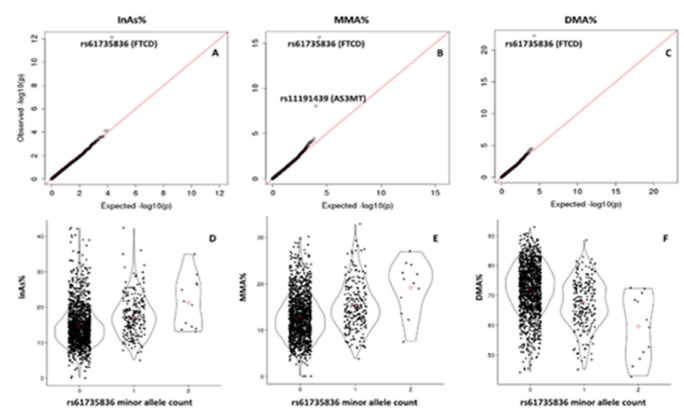

Figure 2. Principal component (PC) scores and correlations between each PC and As species.

and MMA\% $\left(\mathrm{P}=7 \times 10^{-16}\right)$ and decreased DMA $\%$ $\left(\mathrm{P}=8 \times 10^{-22}\right)$ (Fig. 3). Among 2,401 individuals with arsenic-induced skin lesions (an indicator of toxicity and cancer risk) and 2,472 controls, carrying the low-efficiency A allele (frequency $=7 \%$ ) was associated with increased skin lesion risk (odds ratio $=1.35$; $\mathrm{P}=1 \times 10^{-5}$ ). rs61735836 (p.Val101Met) resides in exon 3 of FTCD (formiminotransferase cyclodeaminase) and is in weak linkage disequilibrium with all nearby variants (Pierce et al., submitted, 2018). In exome-wide analyses of all 19,992 post-QC variants, rs61735836 (chr21:47572637 based on hg19) showed a clear association with all three metabolite percentages (Fig. $3 \mathrm{~A}-\mathrm{C}$ ). P-values for this association were $\mathrm{P}=8 \times 10^{-13}$ for $\mathrm{iAs} \%, \mathrm{P}=2 \times 10^{-16}$ for MMA $\%$, and $\mathrm{P}=6 \times 10^{-23}$ for $\mathrm{DMA} \%$. The minor allele (A) was associated with decreased DMA $\%$ and increased MMA\% and iAs\% (Fig. 3 D-F), consistent with the associations previously observed for SNPs in the AS3MT region (Pierce et al., submitted 2018). Together, FTCD and $A S 3 M T$ SNPs explain $\sim 10 \%$ of the variation in DMA\% and support a causal effect of arsenic metabolism efficiency on arsenic toxicity in Bangladesh population. Variants in both loci also show suggestive gene-arsenic interactions Using genomewide SNP variations data among related individuals in our study population, we have previously shown that as high as $61 \%$ of variations in DMA\% could be explained by genetic variations (Gao et al., 2015). We have proposed novel integrated approaches to evaluate subtle, but more pervasive, gene-arsenic interactions beyond these two major loci by using other omics (gene expression and epigenomic) data (Argos et al., 2018).

To identify alterations in genome that are induced by arsenic exposure as an attempt to elucidate potential biological mechanism underlying health effects of arsenic we also evaluated genome-level variations in gene expression and DNA methylation. We identified a number of novel gene expression and DNA methylation alterations that are significantly associated with arsenic exposure (Argos et al. 2015; submitted 2018; Gao et al., 2015). In an independent integrated genomics study, we have recently shown that gene expression and DNA methylation 


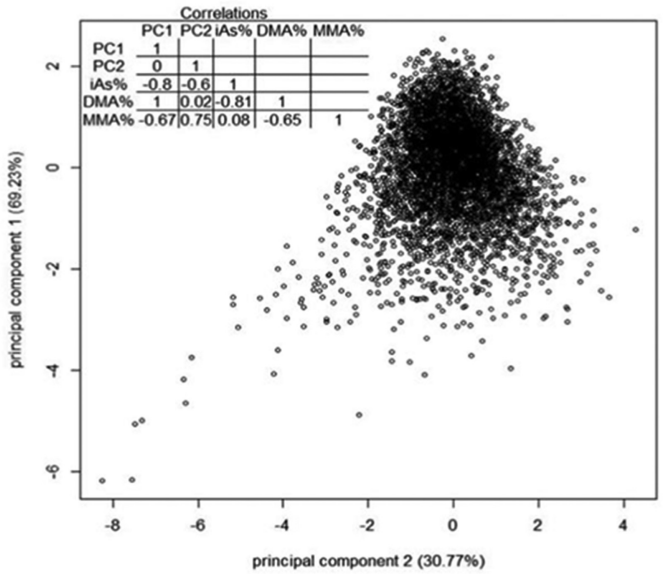

Figure 3. FTCD protein-coding SNP rs61735836 is associated with the all three arsenic species measured in urine.

alterations are co-regulated in the genome (Pierce et al., 2018). Future studies will investigate whether arsenic-induced gene expression and DNA methylation alterations are co-regulated in a manner that can be amenable to interventions.

\section{CONCLUSIONS}

In summary, our body of work implicates two genetic loci, a common variant locus in the $A S 3 M T / \mathrm{C} 10$ orf32 locus at $10 \mathrm{q} 24.32$ and a protein-altering variant in FTCD locus at chr21:47572637 influencing arsenic metabolism efficiency and risk for arsenic-induced skin lesions, the most common sign of arsenic toxicity. We also identified novel genomic alterations potentially induced by arsenic exposure and novel approaches to investigate them. Future studies can use these results, to study the effects of arsenic exposure and metabolism efficiency on health outcomes believed to be affected by arsenic (e.g., cancer and cardiovascular disease), even in the absence of data on arsenic exposure. Knowledge of variation in these regions and associated biological processes could be used to develop intervention and pharmacological strategies aimed at preventing large numbers of arsenic-related deaths in arsenic-exposed populations worldwide.

\section{REFERENCES}

Ahsan, H., Chen, Y., Kibriya, M.G., Slavkovich, V., Parvez, F., Jasmine, F., Gamble, M.V. \& Graziano, J.H. 2007. Arsenic metabolism, genetic susceptibility, and risk of premalignant skin lesions in Bangladesh. Cancer Epidemiol. Biomarkers Prev. 16(6): 1270-1278.
Argos, M., Chen, L., Jasmine, F., Tong, L., Pierce, B.L., Roy, S., Paul-Brutus, R., Gamble, M.V., Harper, K.N., Parvez, F., Rahman, M., Rakibuz-Zaman, M., Slavkovich, V., Baron, J.A., Graziano, J.H., Kibriya, M.G. \& Ahsan, H. 2015. Gene-specific differential DNA methylation and chronic arsenic exposure in an epigenome-wide association study of adults in Bangladesh. Environ. Health Perspect. 123(1): 64-71.

Argos, M., Tong, L., Roy, S., Sabarinathan, M., Ahmed, A., Islam, M.T., Islam, T., Rakibuz-Zaman, M., Sarwar, G., Shahriar, H., Rahman, M., Yunus, M., Graziano, J.H., Jasmine, F., Kibriya, M.G., Zhou, X., Ahsan, H. \& Pierce, B.L. 2018. Screening for gene-environment $(\mathrm{G} \times \mathrm{E})$ interaction using omics data from exposed individuals: an application to gene-arsenic interaction. Mamm. Genome 29(1-2): 101-111.

Farzan, S.F., Karagas, M.R., Jiang, J., Wu, F., Liu, M., Newman, J.D., Jasmine, F., Kibriya, M.G., Paul-Brutus, R., Parvez, F., Argos, M., Scannell Bryan, M., Eunus, M., Ahmed, A., Islam, T., Rakibuz-Zaman, M., Hasan, R., Sarwar, G., Slavkovich, V., Graziano, J., Ahsan, H. \& Chen Y. 2015. Gene-arsenic interaction in longitudinal changes of blood pressure: Findings from the Health Effects of Arsenic Longitudinal Study (HEALS) in Bangladesh. Toxicol. Appl. Pharmacol. 288(1): 95-105.

Gao, J., Tong, L., Argos, M., Scannell Bryan, M., Ahmed, A., Rakibuz-Zaman, M., Kibriya, M.G., Jasmine, F., Slavkovich, V., Graziano, J.H., Ahsan, H. \& Pierce, B.L. 2015. The genetic architecture of arsenic metabolism efficiency: a SNP-based heritability study of Bangladeshi adults. Environ. Health Perspect. 123(10): 985-992.

Gao, J., Roy, S., Tong, L., Argos, M., Jasmine, F., Rahaman, R., Rakibuz-Zaman, M., Parvez, F., Ahmed, A., Hore, S.K., Sarwar, G., Slavkovich, V., Yunus, M., Rahman, M., Baron, J.A., Graziano, J.H., Ahsan, H. \& Pierce, B.L. 2015. Arsenic exposure, telomere length, and expression of telomere-related genes among Bangladeshi individuals. Environ. Res. 136: 462-469.

Jansen, R.J., Argos, M., Tong, L., Li, J., Rakibuz-Zaman, M., Islam, M.T., Slavkovich, V., Ahmed, A., Navas-Acien, A., Parvez, F., Chen, Y., Gamble, M.V., Graziano, J.H., Pierce, B.L. \& Ahsan, H. 2016. Determinants and consequences of arsenic metabolism efficiency among 4,794 individuals: demographics, lifestyle, genetics, and toxicity. Cancer Epidemiol. Biomarkers Prev. 25(2): 381-390.

Pierce, B.L., Kibriya, M.G., Tong, L., Jasmine, F., Argos, M., Roy, S., Paul-Brutus, R., Rahaman, R., RakibuzZaman, M., Parvez, F., Ahmed, A., Quasem, I., Hore, S.K., Alam, S., Islam, T., Slavkovich, V., Gamble, M.V., Yunus, M., Rahman, M., Baron, J.A., Graziano, J.H. \& Ahsan, H. 2012. Genome-wide association study identifies chromosome 10q24.32 variants associated with arsenic metabolism and toxicity phenotypes in Bangladesh. PLoS Genet. 8(2): e1002522. 